\title{
BMJ Open External cold and vibration for pain management of children undergoing needle-related procedures in the emergency department: a randomised controlled non-inferiority trial protocol
}

\author{
Ariane Ballard, ${ }^{1,2}$ Christelle Khadra, ${ }^{1,2}$ Samara Adler, ${ }^{3}$ Evelyne D Trottier, ${ }^{4}$ \\ Benoit Bailey, ${ }^{4}$ Naveen Poonai, ${ }^{5,6,7}$ Jean Théroux, ${ }^{8}$ Sylvie Le May ${ }^{1,2}$
}

To cite: Ballard A, Khadra C Adler S, et al. External cold and vibration for pain management of children undergoing needle-related procedures in the emergency department: a randomised controlled non-inferiority trial protocol. BMJ Open 2019;9:e023214. doi:10.1136/ bmjopen-2018-023214

- Prepublication history for this paper is available online. To view these files, please visit the journal online (http://dx.do org/10.1136/bmjopen-2018023214).

Received 26 March 2018 Revised 28 August 2018 Accepted 20 September 2018

Check for updates

(c) Author(s) (or their employer(s)) 2019. Re-use permitted under CC BY-NC. No commercial re-use. See rights and permissions. Published by BMJ.

For numbered affiliations see end of article.

Correspondence to

Ariane Ballard;

ariane.ballard@hotmail.com

\section{ABSTRACT}

Introduction Needle-related procedures are considered as the most important source of pain and distress in children in hospital settings. Considering the physiological and psychological consequences that could result from these procedures, management of pain and distress through pharmacological and non-pharmacological methods is essential. Therefore, it is important to have interventions that are rapid, easy-to-use and likely to be translated into clinical practice for routine use. The aim of this study will be to determine whether a device combining cold and vibration (Buzzy) is non-inferior to a topical anaesthetic (liposomal lidocaine $4 \%$ cream) for pain management of children undergoing needle-related procedures in the emergency department.

Methods and analysis This study will be a randomised controlled non-inferiority trial comparing the Buzzy device to liposomal lidocaine $4 \%$ cream for needle-related pain management. A total of 346 participants will be randomly assigned in a 1:1 ratio to one of the two study groups. The primary outcome will be the mean difference in pain intensity between groups during needle-related procedures. A noninferiority margin of 0.70 on the Color Analogue Scale will be considered. A Non-inferiority margin of 0.70 on the Color Analogue Scale will be considered. The secondary outcomes will be the level of distress during the procedure, the success of the procedure at first attempt, the occurrence of adverse events, the satisfaction of both interventions and the memory of pain 24 hours after the procedure. The primary outcome will be assessed for non-inferiority and the secondary outcomes for superiority.

Ethics and dissemination This study protocol was reviewed and approved by the institutional review board of the study setting. Findings of this trial will be disseminated via peerreviewed publications and conference presentations. Trial registration number NCT02616419.

\section{INTRODUCTION}

Background and rationale

Needle-related procedures, such as venipunctures and intravenous catheter insertions, are considered as the most important source

\section{Strengths and limitations of this study}

- This is the first study to assess the efficacy of the Buzzy device in Canada.

- The large sample size of 346 participants will provide enough power to demonstrate the non-inferiority of the Buzzy device compared with a topical anaesthetic.

- The non-inferiority margin is justified on both clinical and statistical grounds.

- This study presents potential clinical implications for nursing and medical practices in the emergency department.

- The main limitation of this trial is the impossibility to blind participants and personnel to intervention allocation.

of pain and distress in children in hospital settings. ${ }^{1-4}$ The intensity of pain and distress caused by these procedures can vary from mild to moderate for some, while for others, it may be severe. ${ }^{4-7}$ It is now recognised that even a such minor procedure, can result in numerous physiological, psychological and emotional consequences. ${ }^{8}$ Among these, needle phobia is the most important and prevalent one with more than $60 \%$ of children reporting an extreme fear of needles following a bad needle experience. ${ }^{10}$ These children are more likely to report higher levels of pain and distress from subsequent procedures, ${ }^{11} 12$ and they can experience physiological symptoms, such as vasovagal reactions and an increased heart rate and blood pressure. ${ }^{13} 14$ Furthermore, they can also develop healthcare avoidance behaviours in adulthood, such as delays in care, non-compliance of immunisation requirements and avoidance of treatment. ${ }^{1014}$ Consequently, nurses play a critical role in the assessment and management of 
children's pain and distress, and the use of pharmacological and non-pharmacological interventions must be an integrant part of nursing practice. ${ }^{15}$

Procedural pain management represents a major challenge for nurses, specifically for those working in the emergency department (ED). Consequently, children are at high risk for undertreatment of their pain during needle-related procedures. ${ }^{16}$ Although healthcare professionals recognise the importance of providing adequate procedural pain management, it is still suboptimal. ${ }^{817-19}$ Several studies have identified different barriers to using available pharmacological and non-pharmacological interventions for pain management in the ED. ${ }^{817-19}$ Barriers most frequently identified by nurses are time constraints, heavy workload, staffing limitations, space limitations, lack of knowledge and interruptions in the continuity of care. ${ }^{1520-22}$

Currently, the gold standard intervention for needle-related procedural pain is the application of a topical anaesthetic prior to the procedure and several systematic reviews and meta-analysis have demonstrated its efficacy. ${ }^{23-26}$ However, topical anaesthetics require an application time ranging from $30 \mathrm{~min}$ to $60 \mathrm{~min}$, making their implementation for routine use difficult in the rapid and busy setting of the ED. ${ }^{2728}$ Indeed, a study led by Papa and Zempsky ${ }^{27}$ showed that only $28 \%$ of ED nurses used a topical anaesthetic during painful procedures they reported that main barriers to using it were the onset of action of the drug, treatment delays caused by application time and the vasoconstriction of blood vessels. ${ }^{27}$ Consequently, topical anaesthetics do not seem to be an optimal intervention for procedural pain management in an acute care setting where time constraints represent an important challenge to adequate pain control. ${ }^{21}{ }^{29}$ In addition, they had a minimal side effect profile, including minor local reactions, such as mild irritation, redness, itching, oedema or rash of the skin site following the application in 25\%-50\% of cases. ${ }^{24} 25$ 29-31

Other pharmacological and non-pharmacological interventions have also been evaluated for their efficacy on children's pain management and distress during needle-related procedures. Among these, there are sweettasting solutions, ${ }^{32} 33$ needle-free injection systems, ${ }^{34} 35$ vapocoolant sprays ${ }^{36}$ and distraction. ${ }^{37} 38$ However, even if the efficacy of most of these interventions is well demonstrated, their use remains limited in clinical practice. ${ }^{15}$ 20-22 In fact, these interventions may require specific training for healthcare professionals, preparation time, or excessive cost, which represent barriers to their implementation in the fast-paced environment of the ED setting. ${ }^{15}$ 20-22

The limited applicability of both pharmacological and non-pharmacological interventions to manage procedural pain and distress in the ED setting demonstrates a need for innovation in this domain. The optimal intervention for needle-related procedural pain management in the ED would need to be rapid, easy-to-use and without side effects. To answer this problem, Dr Amy Baxter, an emergency paediatrician and pain researcher in the USA, developed a pain blocker device called Buzzy (MMJ Labs, Atlanta, Georgia, USA) specifically for pain management of children undergoing needle-related procedures. The Buzzy is a bee-shaped device combining vibration (body of the bee) and cold (removable ice wings). ${ }^{39}$ The theoretical bases explaining the action of the device are the Gate Control Theory ${ }^{40}$ and the diffuse noxious inhibitory control theory, which both involve modulation of the transmission of pain. ${ }^{39}$ Therefore, it is theorised that the simultaneous use of vibration and cold would provide optimal pain management.

To date, there have been some randomised controlled trials that have investigated the efficacy of the Buzzy device on pain management in children undergoing needle-related procedures in various medical settings. ${ }^{41-49}$ However, these studies present several limitations such as the absence of an active comparator, ${ }^{4143446-48}$ the lack of prior power analyses or sample size calculation, ${ }^{43}{ }^{44}$ lack or unclear allocation concealment, ${ }^{41-444748}$ among others. Of those studies, only two have been conducted in the ED setting, ${ }^{45}{ }^{49}$ and none have been done in Canada. The Buzzy device seems to be a promising method to reduce and control procedural pain in the ED, and it would be interesting to determine if the it is at least as efficacious as a topical anaesthetic for pain management in children and adolescents during needle-related procedures.

\section{Study objectives}

\section{Primary objective}

To determine if a device combining cold and vibration (Buzzy) is non-inferior (no worse) to a topical anaesthetic (liposomal lidocaine 4\% cream) for pain management in children undergoing needle-related procedures in the ED.

\section{Secondary objectives}

To determine if, in comparison with a topical anaesthetic (liposomal lidocaine 4\% cream), the Buzzy device will:

- Decrease the level of distress during the needle-related procedure.

- Improve the success of the needle-related procedure at first attempt.

- Decrease memory of pain 24 hours after the needle-related procedure.

\section{Other secondary objectives}

- To determine the occurrence of adverse events in each study group.

- To evaluate the satisfaction of parents, children and nurses regarding the use of the Buzzy device and the topical anaesthetic (liposomal lidocaine $4 \%$ cream).

\section{METHOD}

This study protocol was developed in accordance with the Standard Protocol Items: Recommendations for Interventional Trials recommendations. ${ }^{50}$ 


\section{Trial design and study setting}

The study design will be a randomised, controlled, non-inferiority trial with two parallel groups and a 1:1 allocation ratio. This study design is of interest when a new intervention seems to present some advantages over the reference intervention. ${ }^{51}$ Considering that the Buzzy device seems to be less expensive, faster and easier to use than the topical anaesthetic, which is the current reference intervention, the choice of a non-inferiority trial design was justified. As recommended for a non-inferiority trial, ${ }^{51}$ a study demonstrating the superiority of the reference intervention compared with a placebo in a similar context should be used as rationale to support this study design. ${ }^{51}$ For this purpose, the study by Taddio et $a l,{ }^{52}$ which aimed to determine the efficacy of the liposomal lidocaine $4 \%$ cream over a placebo for managing pain resulting from venipuncture in children in the ED, was used as a reference trial to the current study.

This single-centre study will take place in the ED of the CHU Sainte-Justine (Montreal, Quebec, Canada), a university paediatric tertiary hospital centre with a census of more than $80000 \mathrm{ED}$ visits per year.

\section{Participants}

Participants will be deemed eligible if they meet all of the following inclusion criteria: (A) aged between 4 years old and 17 years old, (B) presenting to the ED and requiring a needle-related procedure (venipuncture or intravenous catheter insertion), (C) having the ability to communicate in either French or English and (D) accompanied by at least one parent/legal guardian who can understand, read and speak French or English. We will exclude children with (A) a neurocognitive disability that precludes them from assenting and participating to the study, (B) an inability to self-report pain, (C) a critical or unstable health status $(<3$ on the Canadian Triage and Acuity Scale), (D) a Reynaud's syndrome or sickle cell disease with extreme sensitivity to cold; (E) a break or abrasion on the skin where the device would be installed and (F) a nerve damage or limited sensation in the extremity where the needle-related procedure will be performed. We will not exclude children who received analgesics, including acetaminophen or ibuprofen, within the 4 hours prior to presentation to the ED, but it will be documented.

\section{Interventions}

\section{Experimental group: Buzzy device}

Participants in the experimental group will receive the Buzzy device intervention. The Buzzy is a palm-sized device with two components: (1) body of the bee (vibration) and (2) removable and reusable ice wings (ice). The body of the bee is a vibrating motor powered by two alkaline AAA batteries, and it lasts for about 20 hours. The vibration component is activated by a manual switch on the top part of the device. The removable set of wings contain a total of $18 \mathrm{~g}$ of ice. Each set of ice wings can stay frozen for about $10 \mathrm{~min}$ at room temperature, and they are reusable up to 100 times. Dimensions of the device are $8 \mathrm{~cm} \times 5 \mathrm{~cm} \times 2.5 \mathrm{~cm}$. Enrolled children will have the opportunity to hold and get familiarised with the Buzzy device before the needle-related procedure.

\section{Use and placement of the Buzzy device for the needle-related procedure}

The research nurse will follow the following steps, as recommended by the manufacturer's (MMJ Labs, Atlanta, Georgia, USA): (1) immediately before the needle-related procedure, a set of ice wings will be retrieved directly from the freezer of the ED unit. For optimal efficacy, the wings must be frozen solid; (2) the ice wings will be inserted through the elastic bands fixed on the back of the Buzzy device; (3) when the staff nurse will be ready to clean the site and perform the needle-related procedure, the research nurse will install the Buzzy device on the child's arm, above and as close as possible to the insertion site (about $3-5 \mathrm{~cm}$ ) with a reusable tourniquet, and the vibration will be then activated. The Buzzy device will be installed for about 30 to $60 \mathrm{~s}$ prior to the needle-related procedure; (4) the device will have to be maintained on the child's arm throughout the procedure, at least until the needle is removed; (5) when the procedure is over, the two components of the device will be cleaned with a disinfectant cleaner based on proprietary accelerated hydrogen peroxide (Virox) as per the Infection Prevention and Control guidelines at the study setting; and (6) the ice wings will then be put back in the freezer of the unit for a subsequent procedure.

\section{Physiological basis of the Buzzy device}

The Gate Control Theory ${ }^{40}$ and the Descending Noxious Inhibitory Controls (DNIC) are the theoretical bases of the Buzzy device. More specifically, the Gate Control Theory stipulates that the vibration component of the device blocks the A-delta and $\mathrm{C}$ nociceptive fibres by stimulating the A-beta non-nociceptive fibres. It activates an inhibitory interneuron and results in a reduction of the pain signal transmitted to the spinal cord. ${ }^{49} 53$ The cold component (prolonged cold application 30-60s) stimulates the $\mathrm{C}$ nociceptive fibres and further blocks the A-delta nociceptive pain transmission signal when applied close to the needle insertion site. ${ }^{49}$ The second theory behind the Buzzy device is the DNIC. More specifically, intense cold application stimulates the nociceptive C fibres and activates a supraspinal modulation which, in turn, increases the body's overall pain threshold and therefore produces a generalised hypoalgesia at the insertion site. ${ }^{3954}$

Control group: topical anaesthetic (liposomal lidocaine 4\% cream). Participants in the control group will receive an application of liposomal lidocaine 4\% cream (Maxilene, RGR Pharma, LaSalle, Ontario, Canada) over the insertion site $30 \mathrm{~min}$ before the needle-related procedure. The topical anaesthetic cream will be applied by the research nurse according to the manufacturer's recommendations, and the site will be covered by a Tegaderm dressing 
(3M Canada Company, London, Ontario, Canada). The topical anaesthetic cream and the Tegaderm dressing will be removed just before the procedure. This intervention was chosen as an active control intervention as it has been shown to be the most effective for pain management regarding needle-related procedures, ${ }^{23-26}$ and it is also the standard care currently established in the study setting.

The liposomal lidocaine $4 \%$ cream has been chosen over other topical anaesthetics because of its shorter application time $(30 \mathrm{~min})$ and its minimal vasoactive properties that minimise potential interference with the success of the needle-related procedure.$^{52}$ Currently, the gold standard topical anaesthetic cream is a combination of lidocaine $2.5 \%$ and prilocaine $2.5 \%$ cream (Eutectic Mixture of Local Anesthetics (EMLA)), but it requires an application time of $60 \mathrm{~min}$ and it is frequently associated with vasoconstriction of blood vessels. ${ }^{55-57}$ The liposomal lidocaine $4 \%$ cream has also been chosen due to the lower occurrence of rash reactions after its application, which is often observed with the amethocaine $4 \%$ gel $^{58}$ The ametocaine $4 \%$ gel has also been associated with vasodilatation and a risk of hypersensitivity with repeated use. $^{26}$

\section{Mechanism of action}

The mechanism of action of topical anaesthetics relies on the reversible interruption of nerve conduction near the application site by inhibiting sodium influx through the voltage-gated sodium channels. ${ }^{30}{ }^{59-61}$ This inhibition of sodium influx decreases the ability to generate action potentials decreasing or blocking hereby pain signals conduction. Following the application, a temporary loss of sensation in the limited area of application is produced. ${ }^{6061}$

\section{Study proceedings}

\section{Recruitment}

Eligible participants will be recruited consecutively in the ED by two research nurses during study enrolment hours (approximately 25 hours/weeks, depending on research nurses' availability). Potentially eligible children will be initially assessed on arrival to the ED by triage nurses, staff nurses and physicians. Then, when the treating physician will have determined that a child will require a venipuncture or a catheter intravenous insertion, the research nurse will approach the child and its family to confirm study eligibility per the inclusion and exclusion criteria, to explain the study in greater details and to answer all questions before seeking consent for study participation. Informed written consent will be obtained from parents or legal guardians and assent will be obtained from children over 7 years old. Research nurses will maintain and complete a screening and enrolment log to provide a comprehensive list of all children who were screened for eligibility. Recruited children will be randomly allocated to either the experimental (Buzzy device intervention) or the control group (liposomal lidocaine $4 \%$ cream).
Data collection and outcomes measures

Data collection will start following consent and enrolment. All data will be collected by one of the two research nurses using a paper case report form (CRF) developed and designed for this study. In addition to the primary and secondary outcomes, sociodemographic and clinical data and covariates will be recorded. Data will be collected at different end points: before randomisation (T-0), $5 \mathrm{~min}$ before the needle-related procedure (T-1), during the needle-related procedure (T-2), immediately after the needle-related procedure (T-3) and 24 hours after the needle-related procedure (T-4). Of note, the needle-related procedure will be performed by the staff nurse and not the research nurse.

\section{Sociodemographic and clinical data}

Before randomisation of participants (T-0), sociodemographic and clinical data will be collected by the research nurse. These data will include age, sex, reason for consultation, previous experience(s) of needle-related procedures and analgesia received in the last 4 hours prior the procedure. Contact preference and information will also be obtained for a follow-up 24 hours after the needle-related procedure.

\section{Primary outcome measure}

The primary outcome will be the mean difference in pain scores during the needle-related procedure between groups. It will be assessed immediately after the first needle-related procedure attempt using the Color Analogue Scale (CAS) (T-3). This chosen end point aligns with the recommendations on standard assessment of post-needle pain. ${ }^{38}$ The CAS is a self-report paediatric pain scale consisting of a plastic ruler with a mechanical slider and showing a wedge-shape figure gradually changing in colour from white to red. The white end means 'no pain' and the red end means the 'worst pain'. The reverse side of the scale is numbered from $0 \mathrm{~cm}$ to $10 \mathrm{~cm}$ with 0.25 increments, allowing investigators to quantify children's pain. ${ }^{62-64}$ The CAS has shown excellent psychometric properties in children with acute pain in the ED. ${ }^{6365}$ The child will be shown the side with the wedge-shape figure with the mechanical slider in the middle position and will be asked to move the slider to the place that corresponds to the pain he or she experienced during the needle-relate procedure. The meaning of each anchor will also be explained to the child prior to using the scale. The research nurse will record the corresponding pain score.

\section{Secondary outcomes measures}

The secondary outcomes will be the pain intensity during the needle-related procedure (T-3), the level of distress during the needle-related procedure (T-2, T-3), the success of the procedure at first attempt (T-3), the satisfaction with both interventions (T-3), the occurrence of adverse events and the memory of pain 24 hours after the needle-related procedure (T-4). 


\section{Pain intensity}

Mean difference in procedural pain scores between groups will also be assessed using the Faces Pain Scales - Revised $(\text { FPS-R })^{66}$ immediately after the first needle-related procedure attempt (T-3). This self-report pain scale is the revised version of the original scale previously developed by Bieri et $a l^{67}$ The FPS-R consists of six faces, and each of them represents a greater intensity of pain than the previous one. The face on the far-left shows 'no pain', and the face on the far-right shows 'very much pain'. On the reversed side of the scale, each face is associated with a score ranging from 0 to $10(0,2,4,6,8$ and 10$) .{ }^{66}$ This scale is the most recommended to evaluate procedural pain intensity in children, particularly in children aged from 4 years old to 12 years old. ${ }^{68}$ Immediately after the procedure, the research nurse will ask the child to point the face that shows how much pain he or she felt during the needle-related procedure. The research nurse will document the pain score associated with the face identified by the child.

\section{Level of distress}

Mean differences between groups on distress scores during the needle-related procedure will be assessed using the Procedure Behavior Check List (PBCL) ${ }^{69}$ (T-2) and the Children's Fear Scale (CFS) ${ }^{70}$ (T-3). The PBCL is an observational scale specifically developed to evaluate pain-related fear and anxiety during painful procedures. This scale consists of a checklist with eight behavioural items: muscle tension, screaming, crying, restraint used, pain verbalised, anxiety verbalised, verbal stalling and physical resistance. The observer has to rate the intensity of each behaviour on a scale from 1 to 5 (1=very mild distress; $5=$ extremelyintense distress) ${ }^{69}$ The research nurse will record the PBCL during the first needle-related procedure attempt (T-2). The CFS is a self-report scale developed to measure fear of children during painful experiences. This scale has five faces with a range of scores from 0 to 4 as each face shows an increasing amount of being scared moving from left to right. ${ }^{70}$ Immediately after the first needle-related procedure attempt (T-3), the child will be asked to choose the face that best shows how much he was scared during the procedure. The child will be informed that the first face is 'not scared at all' and the last face is 'the most scare possible'.

\section{Success of the procedure at first attempt}

The proportion of participants achieving a successful procedure at first attempt will be recorded as a binary outcome (yes/no) (T-3). If the procedure is not successful at first attempt, the research nurse will document the number of attempts in the CRF.

\section{Satisfaction}

Satisfaction of both interventions will be evaluated using three questionnaires tailored for children, parents and nurses and including Likert scale questions and dichotomised (yes/no) questions. It will be assessed immediately after the needle-related procedure with parents and children (T-3) and when reaching 50\% of the targeted recruitment for nurses.

\section{Adverse events}

The proportion of participants experiencing an adverse event will be recorded as a binary outcome (yes/no). An adverse event will be defined as an unexpected medical occurrence in a participant that may or may not be necessarily causally related to one of the two interventions. Adverse events will be recorded after enrolment of the participant until hospital discharge.

\section{Memory of pain}

The memory of pain will be assessed by comparing pain scores between groups 24 hours after the needle-related procedure using the FPS-R phrased in terms of recall ${ }^{66}$ (T-4). After the needle-related procedure, the research nurse will give a paper copy of the FPS-R to each parent or legal guardian with the corresponding instructions. They will be informed that they will be contacted in the next 24 hours ( \pm 6 hours) by telephone, text message or email, depending on their preference. The research nurse will then ask the child to point at the face that corresponds with how much pain they remember feeling during the needle-related procedure at the ED. The child/parent will report by telephone, text message or email the chosen face (first, second, third, fourth, fifth and sixth) and the research nurse will record the answer.

\section{Covariates}

Data will be collected from participants and their parents for potential covariates. More specifically, preprocedural pain (CAS) and preprocedural level of distress (PBCL and CFS) will be assessed $5 \mathrm{~min}$ before the needle-related procedure (T-1). Clinical data will be also recorded during the needle-related procedure (T-2), including: type of procedure (venipuncture and intravenous catheter insertion), healthcare professional performing the procedure (nurse, nursing assistant and phlebotomist), presence of parent/legal guardian during the procedure (one parent, two parents and none), position of the child during the procedure (sitting position, on a parent's lap, dorsal decubitus and dorsal decubitus against his will), restraints used during the procedure (yes/no) and use of other non-pharmacological interventions during the procedure.

\section{Data management}

All data collected with the CRFs will be manually entered into an electronic database statistical software, and the original CRFs will be kept on file at the participating site. Data entry and coding will be performed by the same person. A verification will be done by a second person to compare with the original CRFs. Each participant's file will be assigned an identification number to preserve participant confidentiality. Files will be stored in numerical order in a locked file cabinet in the principal investigator's office at the research centre. Files will be maintained in storage for a period of minimum 25 years 
after completion of the study, according to Health Canada regulations for Health Canada Regulated Clinical Trials.

\section{Randomisation and allocation}

An independent biostatistician of the Applied Clinical Research Unit (Unité de Recherche Clinique Appliquée (URCA) ) will generate the sequence of randomisation as per a computer-generated random listing of interventions applying a permuted block design with random blocks stratified by age (4-7 years; $8-12$ years; $13-17$ years). The SAS software V.9.3 will be used to generate the randomisation list using a prespecified seed to ensure reproducibility and proof of random allocation. To ensure concealment, the block size will not be disclosed. The randomisation sequence will be stored at the URCA for the whole duration of the study in order to keep the investigators blinded from the study conditions. Enrolled participants will be randomly assigned, in a 1:1 allocation ratio, to receive either the experimental intervention (Buzzy device intervention) or the control intervention (liposomal lidocaine 4\% cream).

The allocation concealment will be ensured by the use of sequentially numbered, opaque and sealed envelopes previously prepared by the URCA. After the enrolled participant will have completed all baseline measurements, the appropriate numbered envelope will be opened by the research nurse. Each envelope will contain the randomisation number and the allocated intervention.

Due to the major differences between the two interventions in appearance and timing of application, it will not be possible to blind participants, parents, healthcare providers and outcome assessors (research nurses) to the participant's allocation.

\section{Data analyses}

\section{Sample size}

The primary aim of this trial is to demonstrate the non-inferiority of the Buzzy device compared with a topical anaesthetic (liposomal lidocaine $4 \%$ cream) for procedural pain management during needle-related procedures in the ED. To determine the non-inferiority margin, an electronic survey was sent to 34 paediatric emergency physicians working in ED settings from Quebec and Ontario. The following scenario and question were presented: 'You are seeing a fouryear old female requiring an intravenous catheter for drug delivery. You are considering two interventions for pain management during the needle-related procedure: a topical anesthetic application (liposomal lidocaine 4\% cream) or the Buzzy device. You need to assume that both of these interventions have the potential for reducing needle-related pain'. 'What is the greatest difference in mean pain reduction, on a numerical scale from 0 to 10 , between the topical anasthetic (liposomal lidocaine 4\% cream) and the Buzzy device you are willing to accept to routinely adopt the use of the Buzzy device over the topical anesthetic (liposomal lidocaine $4 \%$ cream) for needle-related procedures?'.
Respondents had to choose a difference ranging from 0.1 to 1.5 with 0.1 increments. The mean answer was 0.70 ; consequently, this value was chosen as the non-inferiority margin. Considering that the minimal clinically significant difference on the CAS in children with acute pain is 1.0 on a scale from 0 to $10^{71}$, the choice of a 0.70 non-inferiority margin is considered conservative and insures that a minimally important difference would not be missed. Therefore, a sample size of 346 participants would be necessary to provide the trial with $90 \%$ power to show the non-inferiority of the Buzzy device compared with a topical anaesthetic at a one-sided alpha level of 0.025 with the use of a non-inferiority margin of 0.70 for the per-procedural pain intensity. We anticipate no loss to follow-up considering the short time frame between the intervention and the assessment of the primary outcome. The sample size was calculated using the G*Power software V.3.0.10.

\section{Statistical methods}

The primary analysis was designed to test whether the Buzzy device is non-inferior to a topical anesthetic $(4 \%$ liposomal lidocaine) for procedural pain management during needle-related procedures, as evaluated by calculating the CI for the mean differences in pain score between groups. Non-inferiority would be declared if the upper limit of the two-sided $95 \%$ CI $(1-2 \alpha \times 100 \% \mathrm{CI})$, or equivalently, the upper limit of the one-sided $97.5 \% \mathrm{CI}$, for the between-group difference (experimental group control group) is less than the predetermined non-inferiority margin of $\Delta 0.70$. In this case, the null hypothesis of inferiority will be rejected in favour of the alternative hypothesis of non-inferiority, and the non-inferiority of the Buzzy device over the topical anesthetic (liposomal lidocaine $4 \%$ cream) will be established. A two-sided 95\% CI will be applied, because it will provide additional information if the superiority of the experimental intervention is demonstrated. ${ }^{51}$ In the case where the non-inferiority is met, superiority testing will be performed using a two-sided alpha of 0.05 and in looking if the upper limit of the CI is less than zero. Non-inferiority analysis will be evaluated according to the intention-to-treat principle (primary analysis) as well as to the per-protocol principle (secondary analysis) to examine for consistency and avoid bias. $^{51}$

The secondary analysis was designed to test the superiority of the Buzzy device over the liposomal lidocaine $4 \%$ cream for secondary outcomes. The Student's t-test will be performed to compare the between-group mean differences in preprocedural and procedural distress scores. The memory of pain 24 hours after the needle-related procedure will also be evaluated by the Student's t-test to compare the mean differences in pain scores between the experimental and control groups. The proportion of participants achieving the success of the procedure at first attempt will be calculated in each group and compared using the $\chi^{2}$ test. Descriptive statistics will be used to report data collected on satisfaction, 
as well as sociodemographic and clinical data. Means and SD will be reported for continuous variables and proportions will be calculated for categorical and nominal variables. Potentially relevant preprocedural and procedural variables will be included in covariate model (analysis of covariance) in an attempt to determine predictors of pain scores reduction. All secondary analysis will be carried out according to the intention-to-treat principle. For superiority testing, a $\mathrm{p}$ value $<0.05$ will be considered as indicating statistical significance.

Preplanned subgroups non-inferiority analyses will be carried out for the primary outcomes based on age group (4-7 years vs $8-12$ years vs $13-17$ years). As we will not have the statistical power in each subgroup to conclude to non-inferiority, the results will be considered as exploratory and will primarily serve for hypothesis generation for future studies. Subgroups superiority analyses will be also performed by age group for secondary outcomes. Multiple imputation methods and sensitivity analysis will be used when possible and appropriate to handle the missing data.

No formal interim analysis is planned for this non-inferiority trial for different reasons. First, there is no necessity to conduct interim analysis for futility reasons in non-inferiority trials considering that even if non-inferiority is established before the completion of the trial, the data collection should be pursued in hope of demonstrating superiority. ${ }^{51}$ Second, considering that we do not expect potentially serious adverse events, interim analysis for safety reasons and stoppings rules are not required..$^{50} 71$ There is also no need to implement a data monitoring committee as the known risks are minimal for both interventions. ${ }^{5072} 73$

\section{Patient and public involvement}

Patients and public were not involved in the design, recruitment and conduct of this study.

\section{DISCUSSION}

This study protocol provides the rationale and methods associated with a randomised controlled non-inferiority trial comparing the Buzzy device to a topical anaesthetic with the aim of improving procedural pain and distress management in children undergoing needle-related procedures. To our knowledge, it will be the first study assessing the efficacy of the Buzzy device in Canada in any clinical setting. A systematic review currently in preparation by our team has identified several limitations in the studies previously conducted on the Buzzy device (PROSPERO ID: CRD42017076531). The present study is carefully designed to overcome these limitations and provide rigorous evidence on its efficacy. The large sample size will allow to determine if the Buzzy device is at least as efficacious as the liposomal lidocaine $4 \%$ cream in decreasing procedural pain. Therefore, this study has the potential to improve clinical care and outcomes of children undergoing needle-related procedures in the ED.
More specifically, findings from this trial could potentially prevent pain and distress experienced by children, as well as improve nurses' pain management practices. In addition, this study could determine the efficacy of the Buzzy device intervention across age ranges and developmental differences. If the non-inferiority of the Buzzy is demonstrated, steps will be taken to eventually obtain a Medical Device Licence from Health Canada to make this device available in the EDs across Canada.

This study presents some limitations that are important to recognise. First, considering the nature and the major differences between both interventions, blinding of participants and personnel will not be possible. Consequently, they will be aware of the intervention allocation once the randomised envelop will be opened. This lack of blinding could influence their behaviour and responses to outcomes, particularly subjective ones like pain and distress creating therefore a potential performance bias. ${ }^{74}$ However, the use of an active comparator (anaesthetic) will potentially reduce or overcome this bias. Indeed, a recent study ${ }^{75}$ has demonstrated that randomised controlled trials using an active comparator reported similar expectation ratings from participants between groups. Second, it will not be possible to blind the secondary outcome assessors (research nurses) as it will require observing the participant's behaviour during the procedure. However, the primary outcome assessment will be assessed by self-report, which is considered as a primary source of evidence for paediatric pain intensity. ${ }^{76}$ This could increase the magnitude of the detection bias as pain is a subjective measure. ${ }^{77}$ However, some have argued that self-report assessment could be considered as equivalent to blinding of outcome assessors considering that it is not associated with an overestimated intervention effects, as is the case in psychotherapy meta-analyses. ${ }^{7879}$ Third, we decided to exclude children under the age of 4 years old as the large majority of blood samples of this population are performed via micromethod (blood collected in capillary tubes from finger sticks), and the use of the Buzzy device is not applicable for these cases. The inability of these children to self-report pain was also a reason for exclusion.

Finally, although there is an increase in use and development of pharmacological and non-pharmacological interventions, pain management remains suboptimal. It suggests that evidence is not being translated in clinical practice or that interventions are underused by healthcare providers. ${ }^{38} 80$ Therefore, it is important to provide healthcare professionals with interventions that are likely to be implemented into clinical practice for routine use. The Buzzy device is an easy-to-use and fast intervention that seems to be a promising option in the ED setting.

\section{ETHICS AND DISSEMINATION}

\section{Ethics and safety consideration}

This approval covers the protocol, informed consent forms and the data collection forms. To date, no 
important protocol modification has been made after the initial ethics approval. As recommended by the International Committee of Medical Journal Editors, ${ }^{81}$ this clinical trial was registered in a public trials registry prior to the beginning of the recruitment (ClinicalTrials.gov: NCT02616419). An Investigational Testing Authorization from the Medical Device Bureau of Health Canada was also granted (\#272708). Finally, this study will be conducted in accordance with the principles of the Declaration of Helsinki ${ }^{82}$ and all REB policies and guidelines. Written informed consent will be obtained from parents or legal guardians, and assent will be obtained from children over 7 years old. Consent involve a follow-up 24 hours after the needle-related procedure. Only the principal investigators (AB, CK and SLM) will be given access to the complete final data sets. Other investigators will have access to the complete final data set if a formal request is formulated and approved by the principal investigators.

\section{Dissemination}

The research protocol has been already presented to local clinicians and stakeholders, as well as at national and international conferences. Scientific results will be disseminated at regional, national and international conferences targeting nurses, emergency physicians and paediatric researchers. A manuscript will be submitted to a high impact peer-reviewed journal.

\section{Trial status}

Recruitment for this study is ongoing.

\section{Author affiliations}

${ }^{1}$ Faculty of Nursing, University of Montreal, Montreal, Quebec, Canada

${ }^{2} \mathrm{CHU}$ Sainte-Justine Research Centre, Montreal, Quebec, Canada

${ }^{3}$ Faculty of Medicine, University of Montreal, Montreal, Quebec, Canada

${ }^{4}$ Division of Emergency Medicine, Department of Pediatrics, CHU Sainte-Justine, Montreal, Quebec, Canada

${ }^{5}$ Department of Emergency Medicine, London Health Sciences Centre, London, Ontario, Canada

${ }^{6}$ Department of Pediatrics, Schulich School of Medicine and Dentistry, Western University, London, Ontario, Canada

${ }^{7}$ Children's Health Research Institute, London Health Sciences Centre, London, Ontario, Canada

${ }^{8}$ School of Health Professions, Murdoch University, Murdoch, Western Australia, Australia

Acknowledgements The authors would like to acknowledge the research nurses (Maryse Lagacé and Ramona Cook) who are currently collecting data for this study. They also thank all the recruited and future participants and their families, as well as the ED staff, for their continuous support. They are particularly grateful to Céline Pinard and Jessie Laflamme, two nurses working in the ED, for their collaboration on this project. The authors also want to acknowledge the Applied Clinical Research Unit of the CHU Sainte-Justine, specifically Aude-Christine Guédon for assistance with biostatistical analyses. $A B$ would like to acknowledge the financial support received from the following organisations: Quebec's Healthcare Research Fund, Quebec's Ministry of Higher Education and Quebec Network on Nursing Intervention Research.

Contributors $A B$ conceptualised and designed the study and wrote the first draft of this research protocol. CK, EDT, BB, NP, JT and SL provided feedback to refine the research methodology. SA, EDT and BB contributed to the implementation of the study. SA is involved in the data collection process. All authors read, critically revised and approved the final version of this research protocol.

Funding This work is supported by funds from the Quebec Network on Nursing Intervention Research.
Competing interests None declared.

Patient consent Obtained.

Ethics approval Research Ethics Board (REB) of the CHU Sainte-Justine (\# 2017-1405).

Provenance and peer review Not commissioned; externally peer reviewed.

Open access This is an open access article distributed in accordance with the Creative Commons Attribution Non Commercial (CC BY-NC 4.0) license, which permits others to distribute, remix, adapt, build upon this work non-commercially, and license their derivative works on different terms, provided the original work is properly cited, appropriate credit is given, any changes made indicated, and the use is non-commercial. See: http://creativecommons.org/licenses/by-nc/4.0/.

\section{REFERENCES}

1. Thurgate $\mathrm{C}$, Heppell $\mathrm{S}$. Needle phobia-changing venepuncture practice in ambulatory care. Paediatr Nurs 2005;17:15-18.

2. Leahy S, Kennedy RM, Hesselgrave J, et al. On the front lines: lessons learned in implementing multidisciplinary peripheral venous access pain-management programs in pediatric hospitals. Pediatrics 2008;122 Suppl 3:S161-S170.

3. Jeffs D, Wright C, Scott A, et al. Soft on sticks: an evidence-based practice approach to reduce children's needlestick pain. J Nurs Care Qual 2011;26:208-15

4. Ortiz MI, López-Zarco M, Arreola-Bautista EJ. Procedural pain and anxiety in paediatric patients in a Mexican emergency department. $J$ Adv Nurs 2012;68:2700-9.

5. McMurtry CM, Pillai Riddell R, Taddio A, et al. Far from "Just a Poke": Common painful needle procedures and the development of needle fear. Clin J Pain 2015;31:S3-11.

6. Birnie KA, Chambers CT, Fernandez CV, et al. Hospitalized children continue to report undertreated and preventable pain. Pain Res Manag 2014;19:198-204.

7. Chidambaran V, Sadhasivam S. Pediatric acute and surgical pain management: recent advances and future perspectives. Int Anesthesiol Clin 2012;50:66-82.

8. Young KD. Pediatric procedural pain. Ann Emerg Med 2005;45:160-71.

9. Brennan F, Carr DB, Cousins M. Pain management: a fundamental human right. Anesth Analg 2007;105:205-21.

10. Taddio A, Ipp M, Thivakaran S, et al. Survey of the prevalence of immunization non-compliance due to needle fears in children and adults. Vaccine 2012;30:4807-12.

11. Noel M, Chambers CT, McGrath PJ, et al. The influence of children's pain memories on subsequent pain experience. Pain 2012;153:1563-72.

12. Noel M, McMurtry CM, Chambers CT, et al. Children's memory for painful procedures: the relationship of pain intensity, anxiety, and adult behaviors to subsequent recall. J Pediatr Psychol 2010;35:626-36.

13. Deacon B, Abramowitz J. Fear of needles and vasovagal reactions among phlebotomy patients. J Anxiety Disord 2006;20:946-60.

14. Wright $\mathrm{S}$, Yelland $\mathrm{M}$, Heathcote $\mathrm{K}$, et al. Fear of needles--nature and prevalence in general practice. Aust Fam Physician 2009;38:172-6.

15. Pretorius A, Searle J, Marshall B. Barriers and enablers to emergency department nurses ' management of patients' pain. Pain Manag Nurs 2015;16:372-9.

16. Ali S, Chambers AL, Johnson DW, et al. Paediatric pain management practice and policies across Alberta emergency departments. Paediatr Child Health 2014;19:190-4.

17. MacLean S, Obispo J, Young KD. The gap between pediatric emergency department procedural pain management treatments available and actual practice. Pediatr Emerg Care 2007;23:87-93.

18. Walco GA. Needle pain in children: contextual factors. Pediatrics 2008;122 Suppl 3(Supplement 3):S125-S129.

19. Trottier ED, Ali S, Le May S, et al. Treating and Reducing Anxiety and Pain in the Paediatric Emergency Department: The TRAPPED survey. Paediatr Child Health 2015;20:239-44.

20. Twycross A. Managing pain in children: where to from here? J Clin Nurs 2010;19(15-16):2090-9.

21. Cramton REM, Gruchala NE. Managing procedural pain in pediatric patients. Curr Opin Pediatr 2012;24:530-8.

22. Fein JA, Zempsky WT, Cravero JP, et al. Relief of pain and anxiety in pediatric patients in emergency medical systems. Pediatrics 2012;130:e1391-e1405.

23. Fetzer SJ. Reducing venipuncture and intravenous insertion pain with eutectic mixture of local anesthetic: a meta-analysis. Nurs Res 2002;51:119-24. 
24. Lander JA, Weltman BJ, So SS. EMLA and amethocaine for reduction of children's pain associated with needle insertion. Cochrane Database Syst Rev 2006;3:CD004236.

25. Shah V, Taddio A, Rieder MJ, et al. Effectiveness and tolerability of pharmacologic and combined interventions for reducing injection pain during routine childhood immunizations: systematic review and meta-analyses. Clin Ther 2009;31 Suppl 2(Suppl 2):S104-S151.

26. Taddio A, Gurguis MG, Koren G. Lidocaine-prilocaine cream versus tetracaine gel for procedural pain in children. Ann Pharmacother 2002;36:687-92.

27. Papa A, Zempsky W. Nurse perceptions of the impact of pediatric peripheral venous access pain on nurse and patient satisfaction. Adv Emerg Nurs J 2010;32:226-33.

28. May K, Britt R, Newman MM. Pediatric registered nurse usage and perception of EMLA. J Soc Pediatr Nurs 1999;4:105-11.

29. Al-Qarqaz F, Al-Aboosi M, Al-shiyab D, et al. Using cold air for reducing needle-injection pain. Int J Dermatol 2012;51:848-52.

30. Beaulieu P. La douleur -Guide pharmacologique et thérapeutique. Montréal: Les Presses de l'Université de Montréal, 2013.

31. Brenner SM, Rupp V, Boucher J, et al. A randomized, controlled trial to evaluate topical anesthetic for 15 minutes before venipuncture in pediatrics. Am J Emerg Med 2013;31:20-5.

32. Harrison D, Yamada J, Adams-Webber T, et al. Sweet tasting solutions for reduction of needle-related procedural pain in children aged one to 16 years. Cochrane Database Syst Rev 2015;150.

33. Stevens B, Yamada J, Ohlsson A. Sucrose for analgesia in newborn infants undergoing painful procedures. Cochrane Database Syst Rev 2010;1:CD001069.

34. Zempsky WT, Bean-Lijewski J, Kauffman RE, et al. Needle-free powder lidocaine delivery system provides rapid effective analgesia for venipuncture or cannulation pain in children: randomized, doubleblind Comparison of venipuncture and venous cannulation pain after fast-onset needle-free powder lidocaine or placebo treatment trial. Pediatrics 2008;121:979-87.

35. Zempsky WT, Robbins B, Richards PT, et al. A novel needle-free powder lidocaine delivery system for rapid local analgesia. $J$ Pediatr 2008:152:405-11.

36. Hogan ME, Smart S, Shah V, et al. A systematic review of vapocoolants for reducing pain from venipuncture and venous cannulation in children and adults. J Emerg Med 2014;47:736-49.

37. Chambers CT, Taddio A, Uman LS, et al. Psychological interventions for reducing pain and distress during routine childhood immunizations: A systematic review. Clin Ther 2009;31(Suppl 2):S77-S103

38. Uman LS, Birnie KA, Noel M, et al. Psychological interventions for needle-related procedural pain and distress in children and adolescents. Cochrane Database Syst Rev 2013;76:CD005179.

39. Baxter A, Cohen LL. A randomized controlled trial of a vibrating and cold device for pediatric venipuncture pain relief. Pediatr Emerg Care 2009;25:707.

40. Melzack R, Wall PD. Pain mechanisms: a new theory. Science 1965;150:971-8.

41. Inal S, Kelleci M. Relief of pain during blood specimen collection in pediatric patients. MCN Am J Matern Child Nurs 2012;37:339-45.

42. Inal S, Kelleci M. The effect of external thermomechanical stimulation and distraction on reducing pain experienced by children during blood drawing. Pediatr Emerg Care 2017:1.

43. Canbulat N, Ayhan F, Inal S. Effectiveness of external cold and vibration for procedural pain relief during peripheral intravenous cannulation in pediatric patients. Pain Management Nursing 2015;16:33-9.

44. Canbulat Şahiner N, İnal S, Sevim Akbay A. The effect of combined stimulation of external cold and vibration during immunization on pain and anxiety levels in children. $J$ Perianesth Nurs 2015;30:228-35.

45. Potts DA, Davis KF, Elci OU, et al. A vibrating cold device to reduce pain in the pediatric emergency department: A randomized clinical trial. Pediatr Emerg Care 2017:24.

46. Schreiber S, Cozzi G, Rutigliano R, et al. Analgesia by cooling vibration during venipuncture in children with cognitive impairment. Acta Paediatr 2016;105:e12-e16.

47. Redfern RE, Chen JT, Sibrel S. Effects of thermomechanica stimulation during vaccination on anxiety, pain, and satisfaction in pediatric patients: A randomized controlled trial. J Pediatr Nurs 2018;38:1-7.

48. Moadad N, Kozman K, Shahine R, et al. Distraction using the BUZZY for children during an iv insertion. J Pediatr Nurs 2016;31:64-72.

49. Baxter AL, Cohen LL, McElvery HL, et al. An integration of vibration and cold relieves venipuncture pain in a pediatric emergency department. Pediatr Emerg Care 2011;27:1151-6.
50. Chan A-W, Tetzlaff JM, Gotzsche PC, et al. SPIRIT 2013 explanation and elaboration: guidance for protocols of clinical trials. $B M J$ 2013;346:e7586.

51. Piaggio G, Elbourne DR, Pocock SJ, et al. CONSORT Group. Reporting of noninferiority and equivalence randomized trials: extension of the CONSORT 2010 statement. JAMA 2012;308:2594-604.

52. Taddio A, et al. Liposomal lidocaine to improve procedural success rates and reduce procedural pain among children: a randomized controlled trial. Can Med Assoc J 2005;172:1691-5.

53. Kakigi R, Shibasaki $H$. Mechanisms of pain relief by vibration and movement. J Neurol Neurosurg Psychiatry 1992;55:282-6.

54. Nahra H, Plaghki L. Innocuous skin cooling modulates perception and neurophysiological correlates of brief $\mathrm{CO} 2$ laser stimuli in humans. Eur J Pain 2005:9:521-30.

55. Waldman SD. Eutectic mixture of local anesthetic (EMLA). Pain Digest 1993;3:104-9.

56. Robieux IC, Kumar R, Rhadakrishnan S, et al. The feasibility of using EMLA (eutectic mixture of local anaesthetics) cream in pediatric outpatient clinics. Can J Hosp Pharm 1990;43:235-6.

57. Villada G, Zetlaoui J, Revuz J. Local blanching after epicutaneous application of EMLA cream. A double-blind randomized study among 50 healthy volunteers. Dermatologica 1990;181:38-40.

58. Poonai N, Alawi K, Rieder M, et al. A comparison of amethocaine and liposomal lidocaine cream as a pain reliever before venipuncture in children: a randomized control trial. Pediatr Emerg Care 2012;28:1-8.

59. Zempsky WT. Chapter 47: Topical Anaesthetics and analgesics. In: McGrath BJS PJ, Walker SM, Zempsky WT, eds. Oxford Textbook of Paediatric Pain. Oxford, UK: Oxford University Press, 2014.

60. Cantisani C, Macaluso L, Frascani F, et al. New patents on topical anesthetics. Recent Pat Inflamm Allergy Drug Discov 2014;8:125-31.

61. Kumar M, Chawla R, Goyal M. Topical anesthesia. J Anaesthesiol Clin Pharmacol 2015;31:450-6.

62. McGrath PA, Seifert CE, Speechley KN, et al. A new analogue scale for assessing children's pain: an initial validation study. Pain 1996;64:435-43

63. Tsze DS, von Baeyer CL, Bulloch B, et al. Validation of self-report pain scales in children. Pediatrics 2013;132:e971-e979.

64. von Baeyer CL. Children's self -reports of pain intensity: scale selection, limitations and interpretation. Pain Res Manag 2006;11:157-62.

65. Bulloch B, Tenenbein M. Validation of 2 pain scales for use in the pediatric emergency department. Pediatrics 2002;110:e33.

66. Hicks CL, von Baeyer CL, Spafford PA, et al. The faces pain scalerevised: Toward a common metric in pediatric pain measurement. Pain 2001;93:173-83.

67. Bieri D, Reeve RA, Champion GD, et al. The faces pain scale for the self-assessment of the severity of pain experienced by children: development, initial validation, and preliminary investigation for ratio scale properties. Pain 1990;41:139-50.

68. McGrath PJ, Walco GA, Turk DC, et al. Core outcome domains and measures for pediatric acute and chronic/recurrent pain clinical trials: PedIMMPACT recommendations. J Pain 2008:9:771-83.

69. LeBaron S, Zeltzer L. Assessment of acute pain and anxiety in children and adolescents by self-reports, observer reports, and a behavior checklist. J Consult Clin Psychol 1984:52:729-38.

70. McMurtry CM, Noel M, Chambers CT, et al. Children's fear during procedural pain: preliminary investigation of the Children's Fear Scale. Health Psychol 2011;30:780-8.

71. D'Agostino RB, Massaro JM, Sullivan LM. Non-inferiority trials: design concepts and issues - the encounters of academic consultants in statistics. Stat Med 2003;22:169-86.

72. Sydes MR, Spiegelhalter DJ, Altman DG, et al. Systematic qualitative review of the literature on data monitoring committees for randomized controlled trials. Clin Trials 2004;1:60-79.

73. Lin JY, Lu Y. Establishing a data monitoring committee for clinical trials. Shanghai Arch Psychiatry 2014;26:54-6.

74. Karanicolas PJ, Farrokhyar F, Bhandari M. Practical tips for surgical research: blinding: who, what, when, why, how? Can J Surg 2010;53:345-8.

75. Laird KT, Tanner-Smith EE, Russell AC, et al. Comparative efficacy of psychological therapies for improving mental health and daily functioning in irritable bowel syndrome: A systematic review and meta-analysis. Clin Psychol Rev 2017;51:142-52.

76. Twycross A, Voepel-Lewis T, Vincent C, et al. A debate on the proposition that self-report is the gold standard in assessment of pediatric pain intensity. Clin J Pain 2015;31:707-12.

77. Higgins JPT, Altman DG, Gotzsche PC, et al. The cochrane Collaboration's tool for assessing risk of bias in randomised trials. BMJ 2011;343:d5928. 
78. Cuijpers P, van Straten A, Bohlmeijer E, et al. The effects of psychotherapy for adult depression are overestimated: a meta-analysis of study quality and effect size. Psychol Med 2010;40:211-23.

79. Leichsenring F, Rabung S. Effectiveness of long-term psychodynamic psychotherapy: a meta-analysis. JAMA 2008;300:1551-65.

80. Stevens BJ, Harrison D, Rashotte J, et al. Pain assessment and intensity in hospitalized children in Canada. $J$ Pain 2012;13:857-65.
81. De Angelis $\mathrm{C}$, et al. Clinical trial registration: a statement from the International Committee of Medical Journal Editors. Can Med Assoc J 2004;171:606-7.

82. World Medical Association. World Medical Association Declaration of Helsinki: ethical principles for medical research involving human subjects. JAMA 2013;310:2191-4.

83. Tsze DS, Hirschfeld G, von Baeyer CL, et al. Clinically significant differences in acute pain measured on self-report pain scales in children. Acad Emerg Med 2015;22:415-22. 